\title{
Forecasting the S\&P 500 Index with Circuit Breakers
}

\author{
Arie Harel, Giora Harpaz \\ Baruch College, The City University of New York, New York, USA \\ Email: Giora.Harpaz@baruch.cuny.edu
}

How to cite this paper: Harel, A., \& Harpaz, G. (2020). Forecasting the S\&P 500 Index with Circuit Breakers. Theoretical Economics Letters, 10, 1205-1212.

https://doi.org/10.4236/tel.2020.106072

Received: October 20, 2020

Accepted: November 30, 2020

Published: December 3, 2020

Copyright $\odot 2020$ by author(s) and Scientific Research Publishing Inc. This work is licensed under the Creative Commons Attribution International License (CC BY 4.0).

http://creativecommons.org/licenses/by/4.0/

\begin{abstract}
The purpose of this paper is to develop a Bayesian model of the S\&P 500 stock index in the presence of a circuit breaker rule that would be useful to traders who wish to update positions when information is limited because of a market trading halt. We assume that the market index is distributed by a Poisson process with an unknown parameter. First, using a conjugate Gamma prior probability distribution, we can revise the distribution of the prior distribution, to get an updated Gamma posterior distribution. Second, we calculate the market index's truncated posterior and predictive distributions in the presence of circuit breakers. Third, our predicted index's values (during the activation of the circuit breakers that results in a fifteen-minute trading halt) are demonstrated by numerical examples. Thus, investors would be able to adjust, their long/short positions, when market information is temporarily unavailable.
\end{abstract}

\section{Keywords}

Circuit Breakers, Bayesian Investors, Forecasting Stock Index Levels

\section{Introduction}

Regulators put the first circuit breakers in place following the market crash that occurred on Monday, October 19, 1987 (so-called the Black Monday), when the Dow Jones Industrial Average (DJIA) shed 508 points (22.6\%) in a single day. Suppose that the stock market has a circuit-breaker (SEC Investor Bulletins, 1987), denoted by: $c b$. Once the index level falls short of $c b$, relative to its closing price the day before, the trading is halted for 15 minutes (for levels 1 and 2). The circuit breakers are imposed by the New York Stock Exchange (NYSE) to maintain orderly market behavior. "The equities and options exchanges have procedures for coordinated cross-market trading halts if a severe market price decline reaches levels that may exhaust market liquidity", according to the New York 
Stock Exchange. The market-wide circuit breakers, as they are called, are measured by a single-day decrease in the S\&P 500 index relative to the closing value the day before. There has been a significant amount of research studies of the circuit breakers, some were empirical studies and the rest theoretical ones. The reader is referred to: Ackert, Church and Jayaraman (2001, 2005), Booth and Broussard (1998), Goldstein and Kavajecz (2004), Greenwald and Stein (1991), Kim and Yang (2004), Kim, Yague and Yang (2008), Lauterbach and Ben-Zion (1993), Lee, Ready and Seguin (1994), Ma, Rao, and Sears (1989), Santoni and Liu (1993), Subrahmanyam $(1994,1997)$. However, none of these studies have attempted to forecast the level of the S\&P 500 Index, in the presence of circuit breakers.

Here are the three levels of the circuit breakers for any major stock market index as established by SEC, in 1987 (SEC Investor Bulletins, 1987):

Level 1: A drop of 7\% from the prior day's closing price of the S\&P 500 triggers a 15-minute trading halt. Trading is not halted if the drop occurs at or after 3:25 p.m., Eastern Time. (ET)

Level 2: A drop of $13 \%$ from the prior day's closing price triggers a 15-minute halt. Trading is not halted if the drop occurs at or after 3:25 p.m. ET.

Level 3: A drop of $20 \%$ from the prior day's closing price triggers a halt for the rest of the day, and trading resumes the following day.

Note that since the establishment of the circuit breakers, levels (2 and 3) have never been triggered. The most common circuit breaker is level-1 and we will focus on it, although our model is quite flexible to deal with levels 2 and 3.

Recently, in the month of March 2020, circuit breakers were triggered at the NYSE a few times, (the only times since October 27, 1997), as the Dow Jones Index (DJIA) and the S\&P 500 Index fell more than 7\% at the open, amid the growing global coronavirus pandemic and the tremendous increase in market volatility, as described in the Wall Street Journal (2020)).

The objective of our paper is to develop a Bayesian forecasting model (Lee 2012) of a major market index (i.e., the S\&P 500 Index) in the presence of circuit breakers. We assume that the market index is distributed by a Poisson process with an unknown parameter. Using a conjugate Gamma prior pdf, we can revise the distribution of the unknown parameter, to get an updated Gamma posterior distribution. In Section 2, we present the Bayesian forecasting model. In Section 3 , we calculate the truncated posterior and predictive distributions of the S\&P 500 Index in the presence of circuit breakers, and provides several numerical forecasts. Section 4, discusses the implications of our model for investors.

\section{The Bayesian Forecasting Model}

Let $Y$ be the S\&P 500 Index level (hereafter, the Index level). Note that without loss of generality our model can be applied to any stock index. We assume that the index level (price) has a Poisson probability distribution function (hereafter, pdf) with an unknown and stationary parameter $\theta$ (Lee (2012), Section 3.4, pp. 
102-104)) Thus, the Index's likelihood function is given by,

$$
I(y \mid \theta)=\frac{\theta^{y} \mathrm{e}^{-\theta}}{y !}, \theta>0, \quad y=0,1,2, \cdots
$$

The investor is assumed to combine his a-priori beliefs with the information obtained from the stock market to generate his/her posterior beliefs about the unknown parameter $\theta$, in Equation (1). The prior information of $\theta$ can be based on the investor's past experience; or on any subjective assessment of the unknown parameter $\theta$. However, to provide tractability, it is convenient to assume that the prior pdf of $\theta$ belong to a natural conjugate family. A prior pdf is called a conjugate prior pdf for a given likelihood if the resulting posterior pdf belongs to the same family of distributions as the prior pdf, but with different parameters. The conjugate prior pdf of $\theta$ in our model, would be a gamma with hyper-parameters $\alpha$ and $\beta$, that is,

$$
P_{0}(\theta)=\frac{\theta^{\alpha-1} \mathrm{e}^{-\theta / \beta}}{\Gamma(\alpha) \beta^{\alpha}}, \alpha>0, \beta>0,
$$

where $\Gamma(\alpha)$ is the Gamma function.

For this particular prior distribution, $\alpha / \beta$ is the prior mean. In order to predict the Index level, the investor has to compute the predictive pdf, which is obtained by integrating with respect to $\theta$ the product of the prior pdf in Equation (2) and the likelihood function in Equation (1), to have,

$$
\begin{aligned}
h(w \mid \alpha, \beta) & =\int_{0}^{\infty} l(y \mid \theta) P_{0}(\theta) \mathrm{d} \theta=\int_{0}^{\infty} \frac{\theta^{w+\alpha-1} \mathrm{e}^{-(1+1 / \beta) \theta}}{w ! \Gamma(\alpha) \beta^{\alpha}} \mathrm{d} \theta \\
& =\frac{\Gamma(w+\alpha)}{w ! \Gamma(\alpha) \beta^{\alpha}(1+1 / \beta)^{w+\alpha}}=\left(\begin{array}{c}
w+\alpha-1 \\
\alpha-1
\end{array}\right)\left(\frac{1}{1+\beta}\right)^{\alpha}\left(\frac{\beta}{1+\beta}\right)^{w} .
\end{aligned}
$$

It should be noted that the predictive in Equation (3) is a negative binomial pdf with hyper-parameters $(\alpha, \beta)$.

Once, the index level is revealed in the stock market, the investor can update his/her prior beliefs with respect to $\theta$, via Bayes' theorem, to obtain the posterior distribution of $\theta$ as follows:

$$
P_{1}(\theta \mid y, \alpha, \beta)=\frac{l(w \mid \theta) P_{0}(\theta)}{\int_{0}^{\infty} l(w \mid \theta) P_{0}(\theta) \mathrm{d} \theta}=\frac{\theta^{y+\alpha-1} \mathrm{e}^{-(1+1 / \beta) \theta}(1+1 / \beta)^{y+\alpha}}{\Gamma(w+\alpha)} .
$$

Thus, the first-period posterior of $\theta, P_{1}(\theta \mid y, \alpha, \beta)$ is again a gamma pdf with the hyper-parameters $\alpha_{1}=y+\alpha$ for the shape parameter, and $\beta_{1}=(1+1 / \beta)^{-1}=\beta /(1+\beta)$ for the scale parameter. The first-period expected value of the posterior pdf of $\theta$ is calculated as:

$$
E\left[P_{1}(\theta)\right]=\int_{0}^{\infty} \frac{\theta^{y+\alpha} \mathrm{e}^{-(1+1 / \beta) \theta}(1+1 / \beta)^{y+\alpha}}{\Gamma(y+\alpha)} \mathrm{d} \theta=\frac{(y+\alpha) \beta}{1+\beta} .
$$

Note that for the gamma pdf the expected value of $\theta$ is given by the ratio of the two parameters as, $\alpha_{1} / \beta_{1}=(y+\alpha) \beta /(1+\beta)$.

Once the index level is observed, the first-period predictive pdf $h_{1}$ is given by 
integrating with respect to $\theta$, the product of the posterior pdf in Equation (4) and the likelihood function in Equation (1), to obtain:

$$
\begin{aligned}
h_{1}(w \mid \alpha, \beta, y) & =\int_{0}^{\infty} l(w \mid \theta) P_{1}(\theta) \mathrm{d} \theta \\
& =\frac{(1+1 / \beta)^{y+\alpha}}{y ! \Gamma(y+\alpha)} \int_{0}^{\infty} \theta^{w+y+\alpha-1} \mathrm{e}^{-(2+1 / \beta) \theta} \mathrm{d} \theta \\
& =\frac{(1+1 / \beta)^{y+\alpha} \Gamma(w+y+\alpha)}{w ! \Gamma(y+\alpha)(2+1 / \beta)^{w+y+\alpha}}, w=0,1,2, \cdots .
\end{aligned}
$$

The first-period predictive pdf is again a negative binomial with updated hyper-parameters $y+\alpha$, and $\beta /(1+\beta)$. The first-period predictive mean is given as:

$$
\frac{(y+\alpha) \beta}{1+\beta}=\frac{\beta}{1+\beta} y+\frac{1}{1+\beta} \alpha \beta
$$

It should be noted that the first-period predictive mean is a weighted average of the sample observation $y$ (the index level) and the posterior mean.

\section{The Circuit Breakers and Index Prediction}

In this section we analyze the impact of the Level-1-circuit breakers on the distribution of the S\&P 500 Index (SEC Investor Bulletins, 1987, and the Wall Street Journal (2020). Denote the level of the circuit breaker as: $c b$, which is $7 \%$ below the closing price (or the opening price of the day) of the index in the precious day. Within the 15-minutes halt in trading, investors are blind and cannot observe the index value that would have been realized in the absence of the circuit breaker. Namely, investors, suffer an information loss, because of their inability to observe the real market prices for 15 minutes. Thus, in the presence of a circuit breaker: $c b$, the first-period truncated (at $c b$ ) posterior pdf of $\theta$, can be computed via Bayes' Theorem, as follows:

$$
\begin{aligned}
P_{1}(\theta \mid \alpha, \beta, w \leq c b) & =\frac{\int_{0}^{c b} l(w \mid \theta) P_{0}(\theta) \mathrm{d} w}{\int_{0}^{\infty} \int_{0}^{c b} l(w \mid \theta) P_{0}(\theta) \mathrm{d} w \mathrm{~d} \theta} \\
& =\frac{\int_{0}^{c b} \frac{(1+1 / \beta)^{y+\alpha}}{y ! \Gamma(y+\alpha)} \theta^{w+y+\alpha-1} \mathrm{e}^{-(2+1 / \beta) \theta} \mathrm{d} w}{\int_{0}^{\infty} \int_{0}^{c b} \frac{(1+1 / \beta)^{y+\alpha}}{y ! \Gamma(y+\alpha)} \theta^{w+y+\alpha-1} \mathrm{e}^{-(2+1 / \beta) \theta} \mathrm{d} w \mathrm{~d} \theta} \\
& =\frac{\mathrm{e}^{-(2+1 / \beta) \theta} \theta^{(y+\alpha-1)}\left(\theta^{c b}-1\right)}{\ln (\theta) \int_{0}^{\infty} \frac{\theta^{(y+\alpha-1)}\left(\mathrm{e}^{\ln (\theta) c b}-1\right)}{\mathrm{e}^{(2+1 / \beta) \theta} \ln (\theta)} \mathrm{d} \theta} .
\end{aligned}
$$

In Figure 1, we show the plot of the truncated posterior pdf in Equation (8) for the given parameter values, namely, $(\beta=1, \alpha=4, c b=0.93)$. Note that it is more convenient to fix $y=1$, which can be interpreted as: $100 \%$. Thus, without loss of generality, we can use $y=1$, everywhere in our numerical examples. 


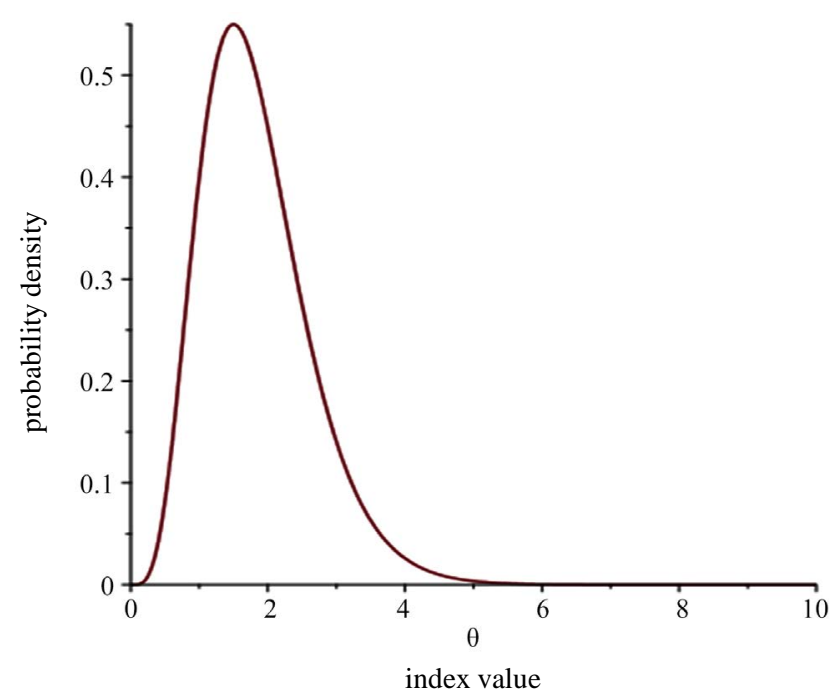

Figure 1. A plot of the truncated posterior pdf of $\theta$, in Equation (8), for selected parameter values the underlying parameters: $\beta=1, \alpha=4, c b=0.93, \quad y=1$.

It is more relevant to derive the predictive pdf of the index value, regardless of the value of the underlying parameter $\theta$. We integrate the product of the likelihood function and the prior pdf, taken into account the truncation of the pdf at the circuit breaker $(c b)$. Thus,

$$
h_{1}(w \mid w \leq c b, \alpha, \beta, y)=\int_{0}^{\infty} \frac{\theta^{w} \mathrm{e}^{-\theta} \mathrm{e}^{-(2+1 / \beta) \theta} \theta^{(y+\alpha-1)}\left(\theta^{c b}-1\right)}{w ! \ln (\theta)\left(\int_{0}^{\infty} \frac{\theta^{(y+\alpha-1)}\left(\mathrm{e}^{\ln (\theta) c b}-1\right)}{\mathrm{e}^{(2+1 / \beta) \theta} \ln (\theta)} \mathrm{d} \theta\right)} \mathrm{d} \theta .
$$

We can also calculate the expected value of the truncated predictive pdf in Equation (9), to derive our predicted index value, in the presence of a circuit breaker $(c b)$ as follows:

$$
\begin{aligned}
& E\left[h_{1}(w \mid w \leq c b, \alpha, \beta, y)\right] \\
& =\int_{0}^{\infty} w\left(\int_{0}^{\infty} \frac{\theta^{w} \mathrm{e}^{-\theta} \mathrm{e}^{-(2+1 / \beta) \theta} \theta^{(y+\alpha-1)}\left(\theta^{c b}-1\right)}{w ! \ln (\theta)\left(\int_{0}^{\infty} \frac{\theta^{(y+\alpha-1)}\left(\mathrm{e}^{\ln (\theta) c b}-1\right)}{\mathrm{e}^{(2+1 / \beta) \theta} \ln (\theta)} \mathrm{d} \theta\right)} \mathrm{d} \theta\right) \mathrm{d} w
\end{aligned}
$$

Equation (10) is quite complicated to compute, in general, but we can calculate the expected value of the truncated predictive pdf, for a few choices of the underlying parameters, alpha and beta. This will generate the expected index value in the presence of a circuit breaker halt. As when the index hits the circuit breaker limit, the trade is halted, and investors don't know what would have been the index value if trading has been continued. There is an information loss to investors created by the presence of the circuit breakers, and the truncation of 
Table 1. The expected value of the truncated predictive pdf from Equation (10).

\begin{tabular}{llllll}
\hline & $\beta=1$ & $\beta=2$ & $\beta=3$ & $\beta=4$ & $\beta=5$ \\
\hline$\alpha=1$ & 0.8549 & 1.0177 & 1.0879 & 1.1270 & 1.1519 \\
$\alpha=2$ & 1.1875 & 1.4185 & 1.5181 & 1.5735 & 1.6089 \\
$\alpha=3$ & 1.5194 & 1.8187 & 1.9476 & 2.0193 & 2.0650 \\
$\alpha=4$ & 1.8518 & 2.2193 & 2.3774 & 2.4654 & 2.5215 \\
$\alpha=5$ & 2.2847 & 2.6204 & 2.8077 & 2.9119 & 2.9783 \\
\hline
\end{tabular}

the predictive pdf in Equation (10). Thus, investors can use the expected truncated price (during the trading halt), in order to predict the market movements, once the exchange would resume the trading. This will allow investors to adjust their trading positions, during the 15-minute trading halt. Only when the trade is resumed, after a 15-minutes halt, investors can observe the true index values.

Table 1 shows the expected value of the truncated predictive pdf from Equation (10), namely, $E\left\lceil h_{1}(w \mid w \leq c b, \alpha, \beta, y)\right\rceil$, for $y=1, c b=0.93$, and for several selected parameter values, $\alpha, \beta, c b$. Note that the Index Value can be scaled by a factor of 1000 to get more realistic predictions.

Thus, the results in Table 1, allow investors to predict the S\&P 500 Index level, (during the 15-minute trading halt) and to adjust, their long/short positions, given these forecasts.

\section{Conclusion}

This paper studies the prediction problem of the Standard \& Poor's 500 Index, in the presence of circuit breakers. Our prediction model would be useful to traders who wish to revise their positions when information is limited due to a trading halt. Our paper has both theoretical and practical applications.

Stock Exchanges in the US instituted the first circuit breakers for trading, after the market crash of October 19th 1987 (so-called the Black Monday), when the Dow Jones Industrial Average (DJIA) shed 508 points (22.6\%) in a single day. In particular, we focus on level-1 circuit breaker that has been triggered a few times in March 2020 (first times since 1997). Thus, a drop of 7\% from the prior day's closing price of the S\&P 500 triggers a 15 -minute trading halt. Trading is not halted if the drop occurs at or after 3:25 p.m., Eastern Time. (ET). In 2020, volatility increased and market-wide circuit breakers were triggered several times.

We developed a Bayesian forecasting model of a major stock index value, in the presence of circuit breakers. It is assumed that the market index (S\&P 500) follows a Poisson distribution with an unknown parameter. Our objective is four-fold. First, using a conjugate Gamma prior distribution, we can revise the distribution of the underlying unknown parameter to obtain an updated Gamma posterior distribution. Second, we calculated the truncated posterior distribution in the presence of a circuit breaker. Third, we calculate the truncated Index's predictive distribution. Note that in the presence of circuit breakers, once they are triggered, investor suffer from information loss for 15 minutes, and they are una- 
ble to view the index value that would have been in the absence of the circuit breaker. Fourth, our truncated predictive distribution provides numerical forecasts of the index value, for a given set of parameters. Our numerical predictions of the truncated expected index value during the activation of the circuit breakers, and the corresponding 15-minute trading halt, allow investors to predict the S\&P 500 Index level, and to adjust, their long/short positions, when market information is temporarily unavailable.

\section{Conflicts of Interest}

The authors declare no conflicts of interest regarding the publication of this paper.

\section{References}

Ackert, L. F., Church, B. K., \& Jayaraman, N. (2001). An Experimental Study of Circuit Breakers: Effects of Mandated Market Closures and Temporary Halts on Market Behavior. Journal of Financial Markets, 4, 185-208.

https://doi.org/10.1016/S1386-4181(00)00020-3

Ackert, L. F., Church, B. K., \& Jayaraman, N. (2005). Circuit Breakers with Uncertainty about the Presence of Informed Agents: I Know What You Know... I Think. Financial Markets, Institutions, and Instruments, 14, 135-168. https://doi.org/10.1111/j.0963-8008.2005.00082.x

Booth, G., \& Broussard, J. P. (1998). Setting NYSE Circuit Breaker Triggers. Journal of Financial Services Research, 13, 187-204. https://doi.org/10.1023/A:1008074725372

Goldstein, M. A., \& Kavajecz, K. A. (2004). Trading Strategies during Circuit Breakers and Extreme Market Movements. Journal of Financial Markets, 7, 301-333. https://doi.org/10.1016/j.finmar.2003.11.003

Greenwald, B. C., \& Stein, J. C. (1991). Transactional Risk, Market Crashes, and the Role of Circuit Breakers. Journal of Business, 64, 443-462. https://doi.org/10.1086/296547

Kim, Y. H., \& Yang, J. J. (2004). What Makes Circuit Breakers Attractive to Financial Markets: A Survey. Financial Markets, Institutions, \& Instruments, 13, 109-146. https://doi.org/10.1111/j.0963-8008.2004.00074.x

Kim, Y. H., Yague, J., \& Yang, J. J. (2008). Relative Performance of Trading Halts and Price Limits: Evidence from the Spanish Stock Exchange. International Review of Economics and Finance, 17, 197-215. https://doi.org/10.1016/j.iref.2007.06.003

Lauterbach, B., \& Ben-Zion, U. (1993). Stock Market Crashes and the Performance of Circuit Breakers: Empirical Evidence. Journal of Finance, 48, 1909-1925.

https://doi.org/10.1111/j.1540-6261.1993.tb05133.x

Lee, C. N. C., Ready, M. J., \& Seguin, P. J. (1994). Volume, Volatility, and New York Stock Exchange Trading Halts. Journal of Finance, 49, 183-214. https://doi.org/10.1111/j.1540-6261.1994.tb04425.x

Lee, P. M. (2012). Bayesian Statistics: An Introduction (4th ed.). Hoboken, NJ: John Wiley and Sons, Ltd.

Ma, C. K., Rao, R. P., \& Sears, R. S. (1989). Limit Moves and Price Resolution: The Case of the Treasury Bond Futures Market. Journal of Futures Markets, 9, 321-335. https://doi.org/10.1002/fut.3990090406

Santoni, G. J., \& Liu, T. (1993). Circuit Breakers and Stock Market Volatility. Journal of 
Futures Markets, 13, 261-277. https://doi.org/10.1002/fut.3990130304

SEC Investor Bulletins (1987).

https://www.sec.gov/oiea/investor-alerts-bulletins/investor-alerts-circuitbreakersbulleti nhtm.html

Subrahmanyam, A. (1994). Circuit Breakers and Market Volatility: A Theoretical Perspective. Journal of Finance, 49, 237-254.

https://doi.org/10.1111/j.1540-6261.1994.tb04427.x

Subrahmanyam, A. (1997). The Ex-Ante Effects of Trade Halting Rules on Informed Trading Strategies and Market Liquidity. Review of Financial Economics, 6, 1-14. https://doi.org/10.1016/S1058-3300(97)90011-2

Wall Street Journal (2020). Circuit Breaker Halts Stock Trading for First Time Since 1997. https://www.wsj.com/articles/traders-closely-watching-circuit-breakers-thresholds-115 83761223 